\title{
Smartphone-Enabled Biotelemetric System for A Smart Contact Lens
}

\author{
George Shaker*, Luyao (Ray) Chen, Safieddin Safavi Naeini \\ University of Waterloo, Waterloo, ON, Canada
}

"Corresponding author: George Shaker, Department of Electrical and Computer Engineering, University of Waterloo, ON, N2L 3G1, Canada. Tel: +15194985173; Email: gshaker@uwaterloo.ca; georgeshaker@ieee.org

Citation: Shaker G, Chen L, Naeini SS (2017) Smartphone-Enabled Biotelemetric System for A Smart Contact Lens. Biosens Bioelectron Open Acc: BBOA-101. DOI: 10.29011/BBOA-101. 100001

Received Date: 11 July, 2017; Accepted Date: 31 July, 2017; Published Date: 05 August, 2017

\begin{abstract}
An all-in-one solution for providing telemetry from a smart contact lens to smartphone is demonstrated here for the first time. This paper discusses how Wi-Fi, Bluetooth, and GSM can be utilized in unison on a modern smartphone to wirelessly power up various microelectronic systems on the contact lens, and to acquire data from the sensors responsible for detecting biomarkers within the tear fluid.
\end{abstract}

Keywords: Biotelemetry; Energy Harvestin; Non-Invasive Diabetes Monitoring; Smart Contact Lenses

\section{Introduction}

A recent surge in technology development dedicated to glucose monitoring and artificial pancreas is both a sign showing fast-increasing public demand for wearable electronics, and the rapid advancement in nanotechnology, material science, and bioelectronics. Finger-pricking has been the only medically accepted glucose monitoring technique for diabetic patients until as recent as 2012, when the US Food and Drug Administration has approved its first Continuous Glucose Monitoring (CGM) system [1], which uses the so called artificial pancreas as an automated closed-loop glucose detection and insulin ad-ministration device that lessens the frequency of daily finger-pricking. However, artificial pancreas also performs glucose analysis invasively through the skin, which is non-ideal or non-feasible in many health monitoring applications. Researchers in both academia and industry are working on non-invasive techniques for monitoring biomarkers, such as glucose, in human sweat and tear $[2,3]$.

These are sought-after biological testbeds for conducting electrochemical measurement in regards to health monitoring. With the advancement in nanotechnology, sensors can be discreetly integrated into skin-like patches, and contact lenses with embedded microelectronics [4]. These microelectronic systems have the advantage of being small, long-lasting, and easily automated with wireless radio technology. There are novel ultra-low energy circuits dedicated for converting glucose concentration in tear fluid to either digital or analog payload $[5,6]$. However, wire- less telemetry of glucose data from the contact lens to an external reader has always been plagued by insufficient power budget due to dimensional constraints of a contact lens, as well as the electromagnetic loss in the environment.

To the authors' knowledge, a realistic and immediately feasible system for the acquisition of data from a contact lens to a smartphone has not been reported. Previously, energy harvesting from unmodulated cellular emission has shown to be feasible for providing sufficient power to microelectronics on the contact lens within an arm's distance [7]. It has also been shown that data can be framed as a standard Bluetooth Low Energy (BLE) payload with very low energy per bit during transmission [8]. Recently, existing wearable devices have been used as RF source and as the receiver to support inter-device backscattering type of radio communication [9]. In this paper, a different approach to utilize backscattering is employed for conveying data directly from the contact lens to a smartphone.

The below figure depicts a scenario where the phone is raised comfortably at a reading distance (Figure 1).

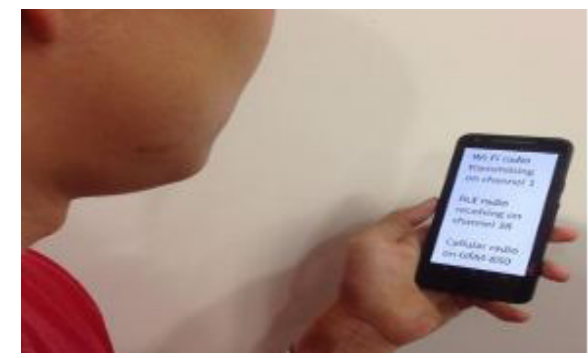

Figure 1: An All-In-One Solution for Enabling a Contact Lens for Glucose Detection on A Smartphone. 
The smartphone's $2 \mathrm{G} / 3 \mathrm{G} / 4 \mathrm{G}$ emissions are utilized as a power source via energy harvesting at the wireless sensing device (contact lens in this case), while a Wi-Fi channel is used as the backscatter source, and a Bluetooth channel to receive the backscattered signals to acquire data from the contact lens.

\section{Theory of Operation}

On the contact lens, the active microelectronic components remain in continuous operation with $\mathrm{DC}$ power rectified from $2 \mathrm{G} / 3 \mathrm{G} / 4 \mathrm{G}$ emissions. Some of the general functions include managing power, sustaining sensor measurement, and generating digital baseband for wireless data transmission. The below figure shows a baseband driving a load modulator, which interfaces with an antenna; the reflective boundary between them allows impinging electromagnetic waves to scatter (Figure 2).
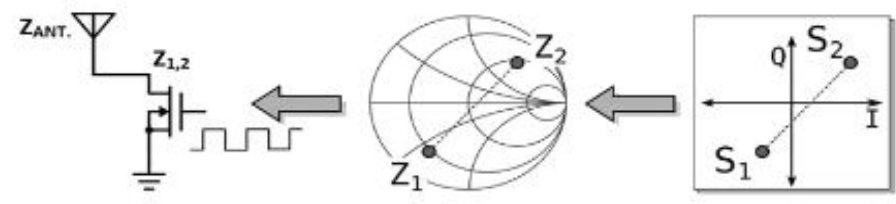

Figure 2: Relationship Between a Signal Constellation and the Load Impedance of the FET [10].

Thus, the glucose payload in baseband becomes a backscattered RF signal. The modulator is made up of a single RF FET, and an antenna connecting to its drain. This enables Binary Frequency Shift Keying (BFSK), where the In-phase and Quadrature components of the BFSK symbols are represented by the drain impedance at the transistor's two regions of operation: Cutoff and Active, which are controlled by voltage levels at the gate. The rapid toggling of the gate is achieved by using digitally generated unipolar square waves. Conveniently, the square waves are separated into their fundamental and harmonic components due to the transistor's nonlinear properties. A demodulator, such as a smartphone, only has to capture the fundamental to recover the original message in the square wave.

A critical caveat is that the BLE baseband, framed with glucose payload, cannot directly be used as the modulating signal, because of the monostatic nature of the backscatter architecture (when the smartphone is used as both the backscatter source and receiver). The high-powered RF source signal would dominate the backscattered signal from the contact lens by orders of magnitude, causing significant self-interference. The solution is to use a subcarrier to bring the BLE baseband frequency to an intermediate frequency, before allowing the signal source from the smartphone to upshift the BLE frame into RF spectrum via backscattering. The intermediate frequency is also generated digitally as unipolar square waves on the contact lens to retain BFSK specification of the baseband. The choice of frequency for the sub-carrier is the primary reason for the involvement of both BLE and Wi-Fi in this backscattering system.
The below figure depicts the channel assignment of the 2.4$\mathrm{GHz}$ ISM band for some of the commonly used BLE and Wi-Fi channels (Figure 3).

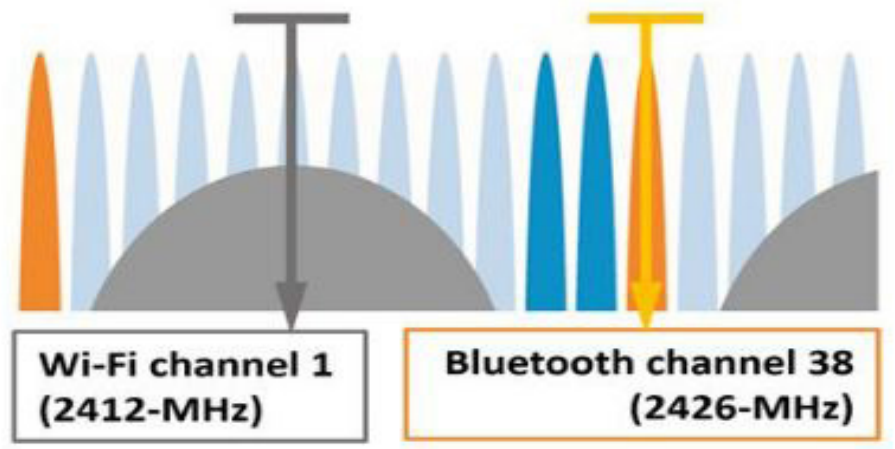

Figure 3: Using Wi-Fi Channel 1 As the RF Source, and Bluetooth Channel 38 As Backscatter Receiver.

By choosing an intermediate frequency of 14- $\mathrm{MHz}$, a deliberate continuous single-tone signal from Wi-Fi channel 1 can be used as an RF source to upshift the BLE frame to an appropriate Bluetooth channel (i.e. advertisement channel 38) for detection. Although Bluetooth and Wi-Fi theoretically can occupy the same spectrum simultaneously, Bluetooth features an adaptive frequency hopping mechanism that avoids occupied frequencies, which allows both protocols to operate concurrently, as long as the assigned channels are vacant

This is also the rationale for choosing the intermediate frequency of 14-MHz, because it avoids overlapping between Bluetooth and Wi-Fi.

\section{Prototyping and Measurement Setup}

The implemented system prototype test-bed validates the theory of operation from the perspective of modulator performance. The below figure shows the system using an Altera Cyclone III FPGA as the unipolar square wave generator and an ATF-55143 RF FET as the modulator - a future implementation will replace these in ASIC on the contact lens (Figure 4).

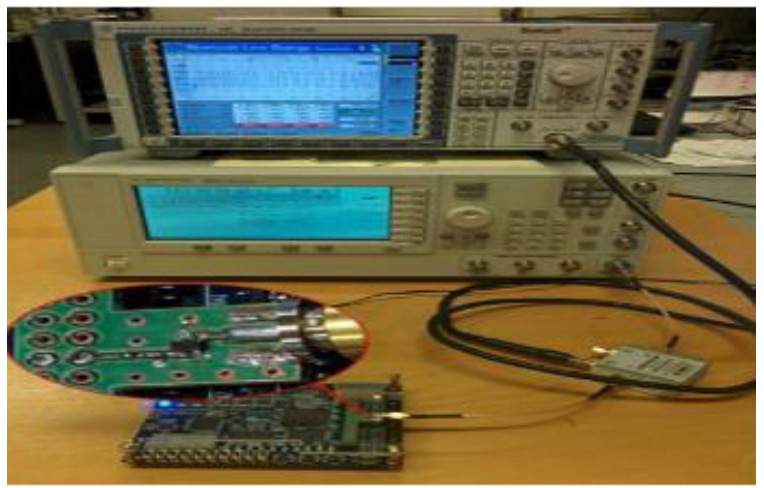

Figure 4: The Backscattering System in Prototype Implementation. 
An Agilent E8257D signal generator is used as the RF source, which will be replaced by Wi-Fi source from a smartphone. A Rohde \& Schwarz CBT is used as the BLE receiver, which will be replaced by a smartphone Bluetooth receiver. The prototype equipment is connected together by common coaxial cables via a Mini-Circuit bi-directional coupler.

The coupler's mainline terminals are its input and output ports, which are connected to the FET modulator and the signal generator, respectively. The coupled forward port is connected to the CBT, while the coupled reverse port is terminated by a 50 load. This configuration allows the power from the signal generator to reach the FET modulator with negligible loss, and also to couple the backscattered signal from the FET modulator to the CBT for detection at a predictable input power. The externally powered FPGA emulates the functionality of digital circuits that will reside in the contact Len's microelectronics, which include the glucose payload, a BLE baseband generator, and an intermediate sub-carrier generator.

\section{Measurement Results}

The Rohde \& Schwarz CBT is an all-around Bluetooth tester. In BLE mode, it has the capability to listen to incoming BLE signals, and to trigger at the rising edge of the burst's zeroth bit. Then, it would be able to perform analysis on the power, modulation, and spectrum behavior of the FET modulator, provided that the received signal has a correct BLE frame on the bit level. A smartphone Bluetooth receiver has the same specifications as the demodulator in the CBT tester.

The below figure shows the first 48 bits of a BLE burst, which are defined by the BLE advertisement frame structure - they are the preamble and access address of a frame (Figure 5).

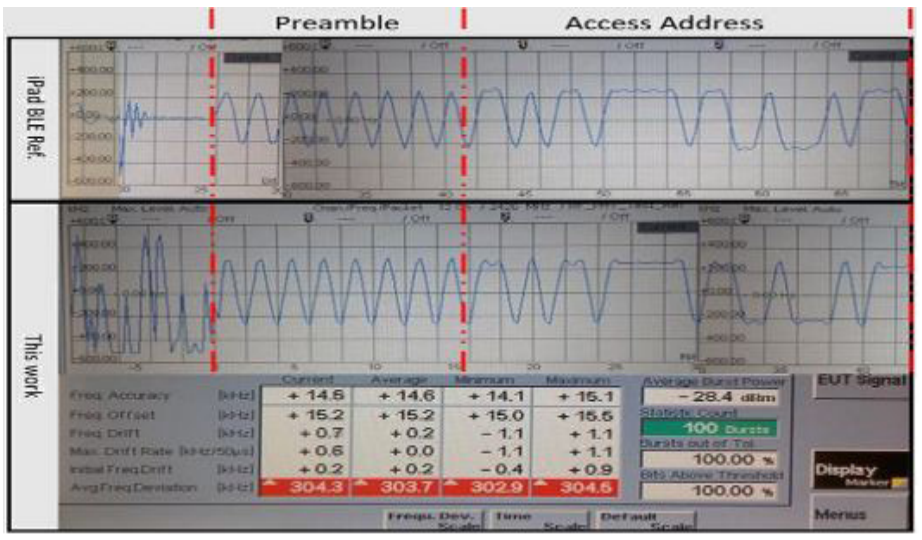

Figure 5: CBT Results of a Demodulated BLE Frame Compared Between Different Sources.

Due to the short time scale, several pictures of the screen are taken at sequential time points of the burst; these pictures are stitched together to display a continuous bit stream. Visually, there is an excellent match when comparison is made between the de- modulated signal emitted from an iPad's iBeacon emulator with that of the backscattered signal from FPGA. The numerical summary of the frequency behavior also indicates a modulator that is within the specifications of commercial BLE devices. The frequency deviation highlighted in red indicates a value above ideal frequency modulation index of 0.45 to 0.55 , but this does not impact the smartphone's ability to demodulate the backscattered signal [8]. There is a loss of approximately $28 \mathrm{~dB}$ to the average power of a backscattered burst signal. This loss is from a combination of absorption at the FET-antenna boundary, nonlinearity of the FET, and the coupling factor of the bi-directional coupler.

\section{Conclusions}

A method of utilizing backscattering by leveraging existing radio resources on modern smartphones is proposed and successfully demonstrated. This technique is used to enable telemetry between smart contact lenses and smartphones for implementing a simple and potentially battery-less glucose detection solution in tear fluid. The backscattering device is a load modulator. The prototype uses a FPGA and a RF FET, while a signal generator and CBT Bluetooth tester are used in place of the smartphone. The backscattered BLE Beacon signal is found to be within the specifications of Bluetooth standards, and the demodulated baseband is nearly identical to the base-band of a standard BLE radio. The below figure shows an energy harvester created for capturing GSM signal on the contact lens, and an eye model with realistic electrical characteristics (Figure 6) [12].

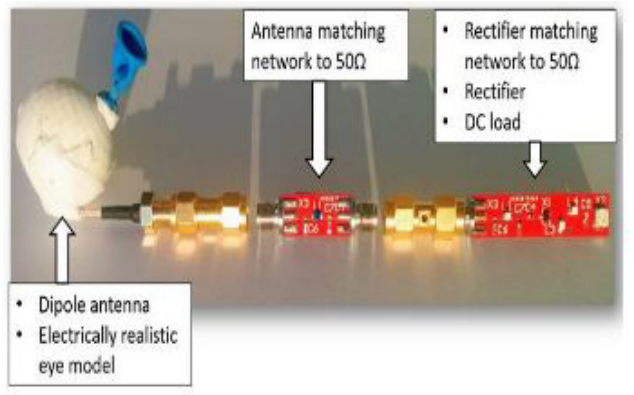

Figure 6: Prototype of a Cellular Emission Energy Harvester Built for Contact Lens Usage.

\section{Acknowledgement}

This work is supported by the National Science and Engineering Research Council of Canada, Medella Health Inc., and the Ontario Centre of Excellence. Special thanks to Lin Li and Luan Le from R\&S for their invaluable support.

\section{References}

1. U.S. Food and Drug Administration. (2012, December). Premarket Approval: DEXCOM G4 Platinum Contiuous Glucose Moni-toring System. DEXCOM, Inc. San Diego, CA, USA. 
Citation: Shaker G, Chen L, Naeini SS (2017) Smartphone-Enabled Biotelemetric System for A Smart Contact Lens. Biosens Bioelectron Open Acc: BBOA-101.

2. Munje RD, Muthukumar S, Prasad S (2017) Lancet-free and label-free diagnostics of glucose in sweat using Zinc Oxide based flexible bioelectronics. Sensors and Actuators B: Chemical 238: 482-490.

3. Yao H, Liao Y, Lingley AR, Fanasiev AA, Lhdesmki I, et al. (2017) A contact lens with integrated telecommunication circuit and sensors for wireless and continuous tear glucose monitoring. Sensors and Actuators B: Chemical 238: 482-490.

4. Vaddiraju S, Burgess DJ, Tomazos I, Jain FC, Papadimitrakopoulos F (2010) Technologies for Continuous Glucose Monitoring: Current Problems and Future Promises. Journal of Diabetes Science and Technology 4: 1540-1562.

5. Dastgheib A and Otis B (2015) Sigma-Delta Analog-To-Digital Converter. JUSTIA patents, USA.

6. Ahmadi MM and Jullien GA (2009) A Wireless-Implantable Microsystem for Continuous Blood Glucose Monitoring. IEEE Transactions on Biomedical Circuits and Systems 3: 169-180.

7. Shaker G, Chen R, Milligan B, Qu T (2016) Ambient electromagnetic energy harvesting system for on-body sensors. IET Electronics Letters 52: 1834-1836.
8. Ensworth JF and Reynolds MS (2015) Every Smart Phone is a Backscatter Reader: Modulated Backscatter Compatibility with Bluetooth 4.0 Low Energy (BLE) Devices. 2015 IEEE International Conference on RFID, San Diego, CA, USA: 78-85.

9. Iyer V, Talla V, Kellogg B, Gollakota S, Smith JR (2016) Inter-Technology Backscatter: Towards Internet Con-nectivity for Implanted Devices. In Proceedings of the 2016 conference on ACM SIGCOMM 2016 Conference, New York, NY, USA: 356-369.

10. Thomas SJ (2013) Modulated Backscatter for Low-Power High-Bandwidth Communication," Ph.D. thesis, Dept. of ECE, Duke University, Durham, USA.

11. Liebl D and Schulz B (2015) 1MA255: LTE and Bluetooth in-device coexistence with WLAN. Rohde \& Schwarz Application Notes.

12. Chen R, Milligan B, Qu T, Shaker G, Naeini SS (2017) Cellular Wireless Energy Harvesting for Smart Contact Lens Applications," IEEE Antennas and Propagation Magazine, in Press. 